\title{
A test bench for small multipolar magnets for a High Intensity superconducting Atomic Beam Source
}

\author{
M. Statera, M. Stancari, V. Carasitti, G. Ciullo, F. Evangelisti, M. Contalbrigo, P. F. Dalpiaz, P. Lenisa
}

\begin{abstract}
The next generation of polarized high energy physics experiments at accelerators employing internal targets requires a significant increase in density of the target. In an atomic beam source (ABS) electron polarization is obtained from SternGerlach separation by sextupole magnets and then transferred to nuclei by means of RF transitions. The Universitá di Ferrara and INFN group is developing a new ABS characterized by an high intensity beam, using small bore $(5-10 \mathrm{~cm})$ high pole tip field $(6-9 T)$ superconducting sextupoles . The $R \& D$ activities performed in Ferrara laboratory regarding a test bench for low temperature field measurement are presented. The system will be built and tested in Ferrara.
\end{abstract}

Index Terms - superconducting multipole magnet, sextupole magnet, field mapping, field harmonics

\section{INTRODUCTION}

Small tapered superconducting sextupole magnets (inner bore $5-10 \mathrm{~cm})$ with an high pole tip field $(6-9 \mathrm{~T})$ are an important part of the design of an high intensity atomic beam source (ABS) characterized by the use of superconducting magnets [1]. The optimization of an high intensity superconducting $\mathrm{ABS}$ requires an high precision sextupoles magnetic field map in order to measure the position of the magnetic focalizing axis and the field distribution in the focalizing volume.

A magnetic field map is a direct measurement of the magnet performances and of the magnet manufacture quality. The presented bench is designed for field mapping of superconducting magnets at $4.2 \mathrm{~K}$ and full current up to $40 \mathrm{~cm}$ long and up to a $34 \mathrm{~cm}$ outer diameter.

At low temperature with the magnet fully powered all the saturation effects, source of uncertainty in simulations, are directly measured. When the saturation effects are important, as in the case of a small sextupole manufactured in Ferrara [2] in which the inner pole was built by iron, and can only be measured in working conditions.

A low temperature field map allow to calculate the maximum pole tip field (extrapolating), to measure the radial field gradient, directly proportional to the focalizing force in an ABS, the magnetic axis and finally the field harmonics.

The pole tip field is the most characterizing single parameter, but has no information about the quality of the focalizing field, such as the focalizing axis respect to the geometrical

Manuscript submitted September 20, 2005

M. Statera, M. Stancari, V. Carasitti, G . Ciullo, F. Evangelisti, M. Contalbrigo, P. F. Dalpiaz, P. Lenisa are with Ferrara University and INFN, Via Sragat 1 corpo C, 44100 Ferrara, Italy axis, or the real radial gradient and the different field harmonics.

In real (not ideal) magnets non ideal fields harmonics are introduced by the non zero assembly tolerances, just like a possible misalignment between the geometrical and magnetic axis.

Sextupoles are used for the focalization of electrically neutral particles because of their particular beam spot geometry reproduction, so any other field component is unlike.

\section{THE BENCH FOR LOW TEMPERATURE MAGNETIC MEASUREMENTS}

There are three typical field measurements of the multipole fields: pole tip field measurement and harmonics measurement by rotating coil or moving hall probes.

The pole tip field measurement can be easily performed during a normal cooldown. For example in the sextupole manufactured in Ferrara, the pole tip field was measured during the training [2].

The rotating coil measurement, consisting in the output reading of the voltage across a coil rotating inside the magnet bore, measure the field harmonics but not the field value.

The moving probe measurement measure the field in a finite number of points inside the magnet bore in a defined direction. Choosing an adequate number of points and measuring directions is possible to attain a good measurement of the field absolute value in a (selected) finite number of points, of its harmonics, its gradient, and the exact position of the zero field (focalizing) axis.

The latter method requires a bigger effort in terms of costs and manpower, but the resulting measurements is much more complete to characterize the multipoles used for the design of a high performance superconducting ABS.

The field mapping bench is realized modifying a top access cryostat (fig. 1): a measuring chamber kept in low vacuum is created to access the magnet's inner bore. Nine hall probes are fixed to a plate connected to a $1.5 \mathrm{~m}$ shaft connected to the movement system. The movement system is in placed on top of the cryostat in air at room temperature.

\section{A. the measurement}

A sextupolar magnet field can be expressed in cylindrical coordinates, neglecting the axial component as a standard 
practice for accelerator magnets, as a function of the radius $r$ and the azimuthal angle $\theta$. Inside the magnet bore the field can be expressed as Fourier expansion both for radial and tangential components:

$$
\begin{gathered}
B_{r}=\sum_{n=1}^{\infty}\left(\frac{r}{R_{r e f}}\right)^{(n-1)}\left[B_{n} \operatorname{sinn} \theta+A_{n} \cos (n \theta)\right] \\
B_{\theta}=\sum_{n=1}^{\infty}\left(\frac{r}{R_{r e f}}\right)^{(n-1)}\left[B_{n} \cos (n \theta)-A_{n} \sin (n \theta)\right]
\end{gathered}
$$

where $A_{n}$ and $B_{n}$ are respectively normal and skew $2 n$-pole components, while $R_{\text {ref }}$ is a reference radius.

The field mapping is performed by ten CMI Hall probes positioned during the measurement with a maximum error of $1 \mathrm{~mm}$. Their active area is a surface of $1.0 \mathrm{~mm}^{2}$ and the magnetic field measurement error is below $3 \%$ for a magnetic field flux between -15 and $15 \mathrm{~T}$. The probes are mounted on the support with a $90^{\circ}$ angle and all of them measure the field in the direction of one of the axis. The probes are mounted on the probe holder shown in fig 4; the ones measuring radial field are placed on the geometrical axis and at $\pm 20 \pm 30 \pm 40 \mathrm{~mm}$ from the axis, while the probes measuring the tangential field are placed on only one side.

The probes are fixed to the holder by screwed wedge to reduce probes misalignment and movements during assembling and measuring.

Rotating the support with $60^{\circ}$ steps the field is measured in the maximum sextupolar radial and tangential components always with the optimal orientation.

This kind of measurement, using the data from all the six $60^{\circ}$ rotations, can measure the Fourier fields components [3] up to 12-pole component. The ratio $\frac{A_{3}}{A_{n}}$ is measured with an error less then $10^{-2}$ for each plane. The field is measured on planes orthogonal to the magnet's geometrical axis with a vertical $1 \mathrm{~cm}$ step. On each plane are then measured 54 points.

The whole measurement is driven by a LabView program, which controls two motors to move the probe holder and acquires the signal from the Hall probes. The data are written on a file, which is analyzed offline.

Each probes reading has two steps: first positioning $(\sim 15$ seconds) and then measuring. The measurement is not performed during a continuous movement in order to reduce the problems in synchronization between motors and data acquisition and take rid of mechanical oscillations during the positioning. The measurement lasts one hour thanks to the fully automated movement. This feature allow to reduce the accelerations by the motors and, with a positioning time of 15 seconds, take rid of the holder oscillations during probe acquisition.

\section{B. The cryostat}

The cryostat used for the test (fig. (1)) is a helium $60 l$ bath one kept at ambient atmosphere designed to test small

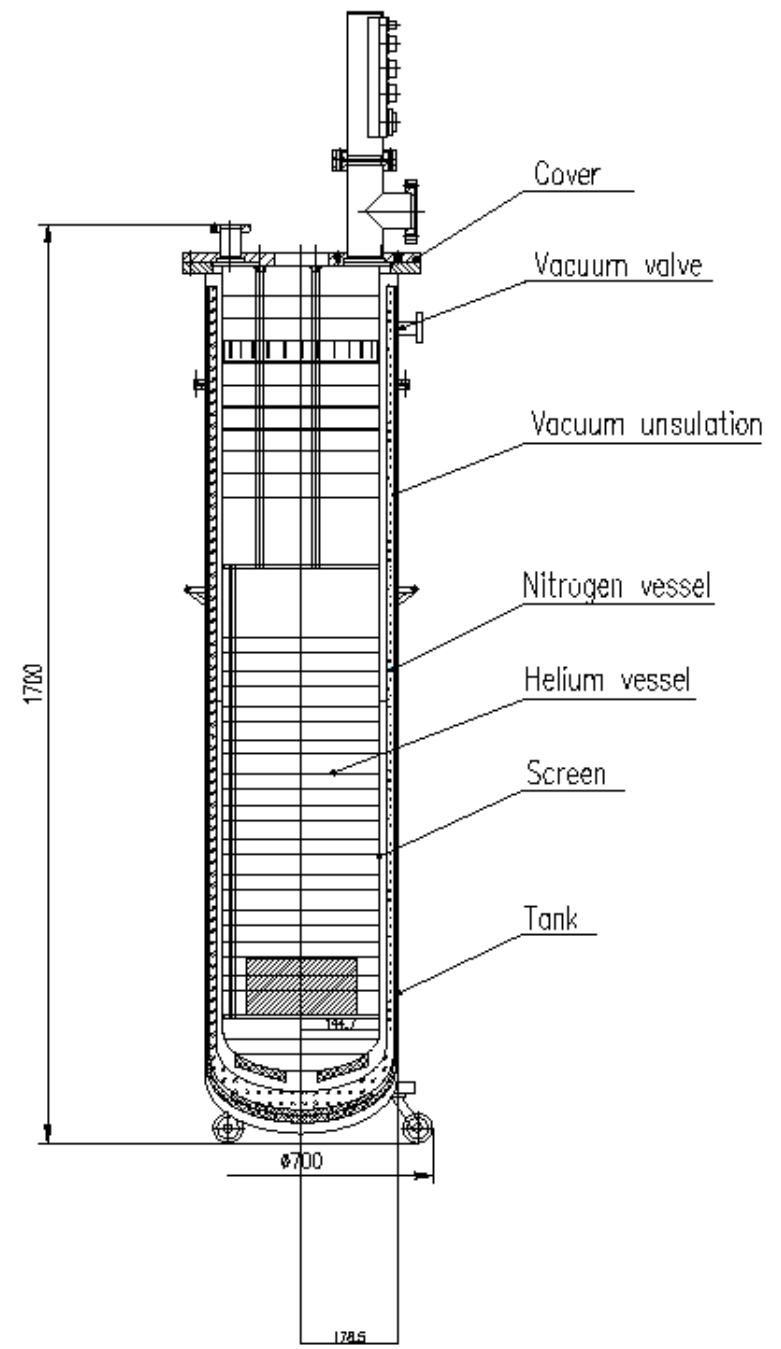

Fig. 1. Cryostat layout before the modification. The helium is kept at $4.2 \mathrm{~K}$ (1 atm). The top flange is thermally insulated by mylar aluminum plates. On the top flange is visible the CF100 flange where the measuring chamber is connected. The magnet is connected to the an intermediate plate by 3 bars (one is shown).

multipoles magnets.

The lateral and bottom thermal insulation is a standard double vacuum layer with a liquid nitrogen vessel between inner $(4.2 \mathrm{~K})$ vessel and the outer room temperature one.

The cold mass and the top thermal insulation are fixed to the top flange. The top thermal insulation is build by seven mylar alumined plates and and intermediate copper plate in good thermal contact with the $77 \mathrm{~K}$ nitrogen vessel. The 25 liters of liquid nitrogen is poured inside the vessel by an inlet on the top of the cryostat.

The cryostat performances where tested in Ferrara [2], the static helium consumption with no cold mass is $0.4 l / h$ and $2.7 l / h$ with a magnet inside.

Three stainless steel bars fixed to the top flange hold the magnet to be tested inside the cryostat. These are used also for the correct positioning of the magnet. 


\section{C. cryostat modification}

The movement for the probes holder must be at room temperature, so a rigid structure is fixed on the top of the cryostat. A plate is moved by one of the two motors. On the movable plate is fixed a second motor with rotate a $2 \mathrm{~m}$ stainless steel hollow shaft to which is fixed the probe holder. The materials entering the magnetic field must have negligible magnetic behavior, in order to avoid any magnetic force on parts nor any magnetic field shielding that could decrease the measurement precision. The allowed materials have magnetic permeability $\mu$ below 1.005 like stainless steel AISI 316LN, Aluminum $\left(1-\mu=3.510^{-4}\right)$ or copper $\left(1-\mu=4.510^{-4}\right)$. The magnet inner bore is accessed by a $4 \mathrm{~cm}$ diameter stainless steel pipe fixed on a CF100 flange on the cryostat top flange and ending in a stainless steel small chamber.

The probe holder is inside the bottom small chamber. A scheme of the measurement apparatus is sketched in fig.2.

The vessel containing the shaft is under low vacuum. Main reason is to avoid ice formation on the inner walls. The vessel is in contact with liquid helium, so that the wall temperature is $4.2 \mathrm{~K}$ at the bottom and room temperature at the upper flange. Ice formation reduces the available space for the measurement and the movement of the shaft.

The small amount of space available, especially inside the magnet's inner bore forced to use a thin stainless steel wall vessel instead of any other insulation.

The time required for a measurement is an important parameter since the inner vessel decrease the total amount of liquid helium inside the cryostat, reducing the the time available for the measurement to 2 hours.

The introduction of the measuring pipe reduces both the liquid helium reserve and the operating time.

The helium capacity of the unmodified cryostat is $60 \mathrm{l}$. The magnet, to be kpt at $4.2 \mathrm{~K}$, must be completely inside the bath and an amount of helium is required to avoid that in case of a quench, the the helium bath does not cover the magnet. The average helium consumption for the tested sextupole magnet is $5.5 \mathrm{l}$. The helium vessel inner diameter is $29.7 \mathrm{~cm}$. The sextupole height is $23.0 \mathrm{~cm}$, considering an overall $30 \mathrm{~cm}$ occupied, there are $34 \mathrm{l}$ of helium available for the measurement.

The liters of helium available after the modification are 33. A small pipe diameter compared to the cryostat inner diameter was chosen to reduce heat flux into the helium bath and decrease as much as possible the reduction of liquid helium available for running the magnet. The small diameter reduces the pumping down conductance.

The system entering the cryostat and the helium bath is kept at low pressure ( $p \sim 1.010^{-2}$ mbar $)$ with helium inside to avoid the risk of ice formation on the walls of the measuring chamber. The low pressure reduces strongly the heat flux due to conduction through gas.

The pressure inside the measuring chamber is strongly reduced by cryopumping when the cryostat is cold, the lower part is infarct at direct contact with liquid helium.

The measuring chamber introduce a heat flux due to conduction through pipes' stainless steel residual gas and probes wires. The heat conduction through metals is easily estimated by Fourier law $\dot{Q}_{C S S}=-\lambda_{1}^{2} A \frac{\Delta T}{l}$, where $A$ is the conducting cross section, $\lambda_{1}^{2}$ the thermal conductivity integral between the temperatures $T_{1}$ and $T_{2}$ at the extremes, $\Delta T=T_{2}-T_{1}$ and $l$ the length of conduction.

The stainless steel thermal conductivity integrated from $4.2 \mathrm{~K}$ and $300 \mathrm{~K}$ is $10.3 \frac{\mathrm{W}}{\mathrm{mK}}$. The worst condition for the outer pipe connecting room temperature with liquid helium is with the bath at maximum level, in that case the conducting length is $1 \mathrm{~m}$, giving a heat flux of $\dot{Q}_{C S S}=0.94 \mathrm{~W}$.

The heat conducted by the helium gas can be estimated [4] by the

$$
\dot{Q}_{C H e}=A_{0} a_{0} P_{m m H g}\left(T_{2}-T_{1}\right) A_{c m^{2}}
$$

where $A_{0}$ is a constant ( 0.0028 for helium), $a_{0}=\frac{a}{2-a}$ is the accommodation coefficient in the case of same area faced surfaces and that each surface has the same accommodation coefficient, $a \leq 0.5$ for helium and we consider the worst case $(a=0.5)$ for a safe calculation, $P_{m m H g}$ is the pressure in torrs, when the surfaces are cold, the pressure at the bottom of the measuring chamber is much below the used pressure again for a safe calculation of $10^{-2} \operatorname{mbar}, T_{2}-T_{1}$ is the temperature difference between the two surfaces $(\sim 300 \mathrm{~K})$ and $A_{\mathrm{cm}^{2}}$ the area in squared centimeters. The total heat flux due to conduction is then

$$
\dot{Q}=\dot{Q}_{C S S}+\dot{Q}_{C H e}=0.94+0.69=1.63 \mathrm{~W}
$$

Corresponding to $2.3 \mathrm{l} / \mathrm{h}$ ofl iquid helium.

\section{D. $D A Q$}

The DAQ system based on LabView. The program controls the magnet temperature, the supplied current, a fast software quench detection, the movement of the Hall probes holder and reads the Hall probes measurement.

The magnet's current is controlled by a feedback loop with the current supplier. There are two quench detection system; the main one is hardware and the software one, reading voltage over each single magnet coil. The measurement is driven by the program starting from the parameters given to the fully programmable motors for the movement to the probes reading and data logging.

This provides a fast, safe, reliable measurement. The program synchronizes the movement and the data acquisition reducing dead times.

The field mapping lasts one hour and is completely automated.

\section{CONCLUSION}

A bench for precise fast and reliable multipolar mapping was presented. The bench allow to map the field of small superconducting multipole at low temperature and full current (actually up to $600 \mathrm{~A}$ ) measuring the field values, field harmonics both normal and skew at least up to 12th component and the position of the magnetic axis. These parameters are fundamental for magnets for a focalizing system for atoms. 


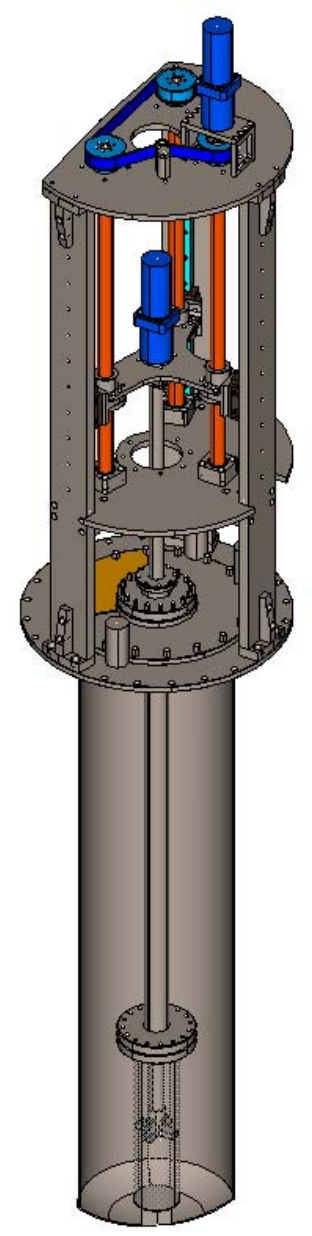

Fig. 2. Overview of the liquid helium vessel, cryostat's top flange and movement system. The shaft entering the measuring chamber and the Hall probes holder are visible.

\section{REFERENCES}

[1] M. s. M. Statera, "A high intensity superconducting atomic beam source," in IEE conf. proc., 2005.

[2] R. F. Michelle Stancari, Marco Statera, "Measurement of a superconducting magnet," INFN, Tech. Rep. INFN-FE-, 2002.

[3] M. C. G. C. M. C. P. F. D. D. R. M. Statera, M. Stancari, "Sextupole room temperature field measurement," Tech. Rep.

[4] experimental techniques in low temperature physics. Oxford, 1979.

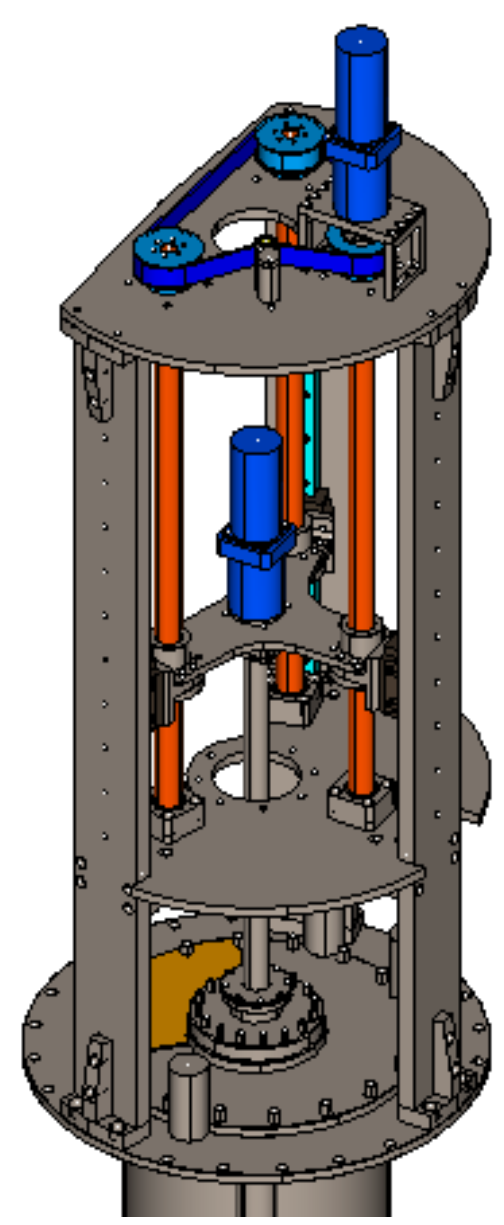

Fig. 3. Top cryostat's flange and precision movement system. In light blue the slits and in orange the recirculating spheres screws. All the drawn parts outside the cryostat are in aluminum. The central plate is vertically movable, the shaft entering the vacuum is rotated by the blue motor on that plate.

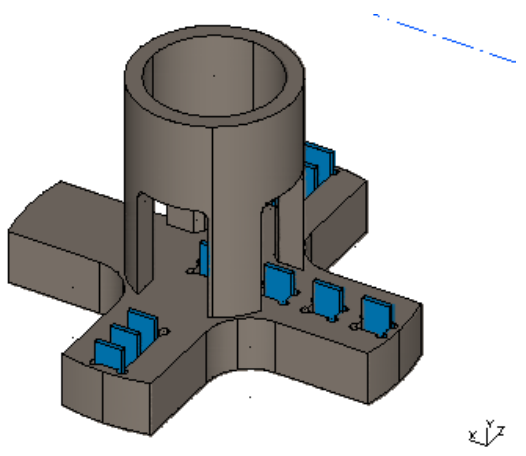

Fig. 4. The probe holder (cross shaped and the connection to the shaft. The Hall probes are blue (real dimension). They are fixed to the holder by screwed wedges.) 